\title{
Repurposing a clinically approved prescription Colquhounia root tablet to treat diabetic kidney disease via suppressing PI3K/ AKT/NF-kB activation
}

\author{
Zhaochen Ma, Yudong Liu, Congchong Li, Yanqiong Zhang ${ }^{*}$ and Na Lin ${ }^{*}$
}

\begin{abstract}
Background: Growing clinical evidences show the potentials of Colquhounia root tablet (CRT) in alleviating diabetic kidney disease (DKD). However, its pharmacological properties and underlying mechanisms remain unclear.

Methods: 'Drug target-Disease gene' interaction network was constructed and the candidate network targets were screened through evaluating node genes' topological importance. Then, a DKD rat model induced by high-fat diet/ streptozotocin was established and used to determine pharmacological effects and network regulatory mechanisms of CRT against DKD, which were also verified using HK2 cell model induced by high glucose.

Results: The candidate network targets of CRT against DKD were involved into various type II diabetes-related and nephropathy-related pathways. Due to the topological importance of the candidate network targets and the important role of the imbalance between immunity and inflammation in the pathogenesis of DKD, PI3K/AKT/NF-KB signaling-mediated immune-modulatory and anti-inflammatory actions of CRT were selected to be experimentally verified. On the basis of high-fat diet (HFD) / streptozotocin (STZ)-induced DKD rat model, CRT effectively reduced the elevated level of blood glucose, decreased the accumulation of renal lipid, suppressed inflammation and the generation of ECM proteins, and ameliorated kidney function and the renal histopathology through inhibiting the activation of PI3K, AKT and NF-KB proteins, reducing the nuclear accumulation of NF-KB protein and the serum levels of downstream cytokines, which were in line with the in vitro findings.
\end{abstract}

Conclusions: Our data suggest that CRT may be the promising candidate drug for treating DKD via reversing the imbalance of immune-inflammation system mediated by the PI3K/AKT/NF-KB/LL-1 $\beta / T N F-a$ signaling.

Keywords: Tripterygium wilfordii Hook F-based therapeutics, Colquhounia root tablet, Diabetic kidney disease, Imbalance of immune-inflammation system, Network pharmacology

*Correspondence: yqzhang@icmm.ac.cn; nlin@icmm.ac.cn Key Laboratory of Beijing for Identification and Safety Evaluation of Chinese Medicine, Institute of Chinese Materia Medica, China Academy of Chinese Medical Sciences, No. 16, Nanxiaojie, Dongzhimennei, Beijing 100700, China

\section{Background}

Diabetic kidney disease (DKD) is a common and severe microvascular complication caused by diabetes. According to the United States Renal Data System, DKD accounts for up to $50 \%$ of end-stage renal disease (ESRD) cases [1, 2], and also the main risk factor for death of patients with diabetes [3, 4]. It is characterized by hyperglycemia, persistent proteinuria, hypertension 
and impaired renal function [5-8]. The main therapeutic strategy of the early DKD is hypoglycemic and antihypertensive combined symptomatic treatment, and the main therapeutic strategy of the late DKD is kidney transplantation. Unfortunately, the efficacy of the current therapeutic strategies is not very satisfying [9]. Although DKD was traditionally indicated as a non-inflammatory glomerular disease caused by metabolic and hemodynamic changes, growing clinical and experimental evidences show that the imbalance between immunity and inflammation may also play an important role in the pathogenesis of DKD [9]. However, there are still few clinical trials on immune-modulatory and/or anti-inflammatory therapeutics [10-12].

Tripterygium wilfordii Hook F. (TwHF) has significant anti-inflammatory and immunosuppressive effects, and is used in the treatment of a wide spectrum of autoimmune disorders and inflammatory diseases with favorable therapeutic efficacy for a long time in China [13, 14]. Among various TwHF-based therapeutics, Colquhounia root tablets (CRT) has been approved by National Medical Products Administration for the treatment of rheumatoid arthritis and systemic lupus erythematosus because of its anti-inflammatory, immunosuppressive and other hormone-like effects. Growing clinical evidences based on relative small samples also show the potentials of CRT in alleviating DKD [15-17]. Especially, Li et al. reported that the clinical effective rate of CRT in the treatment of 70 cases of DKD with massive albuminuria was $94.3 \%$ [18].

Since the scientific evidences confirming the efficacy of CRT in treating DKD is still inadequate and the necessity of exploring new drugs to attenuate renal inflammation, the aim of the current study was to investigate the main pharmacological properties and the underlying molecular mechanisms of CRT against DKD using an integrated network-based computational and experimental strategy.

\section{Materials and methods \\ Data collection and network investigation Prediction of CRT putative targets}

The chemical components containing CRT were collected from our TCM-related database, ETCM (http:// www.tcmip. cn/ETCM/index.php/Home/Index/, Released in 2018) [14]. Then, we chose the chemical components with moderate to good drug-likeness by calculating the quantitative estimate of drug-likeness (QED) score based on models in the Pipeline Pilot ADMET collection as the candidate bioactive components of CRT (QED > 0.49) for the following target predictions. The putative targets hit the candidate bioactive components of CRT were predicted using MedChem Studio (version 3.0; SimulationsPlus, Lancaster, CA, USA, 2012) according to the structural and functional similarities of drugs. The component-putative target pairs with similarity scores higher than 0.80 (high similarity) were selected for further investigation.

\section{Collection of DKD-related genes}

Known therapeutic targets of DKD were collected from the DrugBank database (http://www.drugbank.ca/, version: 5.1.8). Only disease genes that belong to human genes in FDA-approved DKD therapeutic drug targets can be included. In addition, the DKD-related genes were also collected from HPO (The Human Phenotype Ontology, https://hpo.jax.org/app/, last updated on June 8, 2021) and DisGeNet (http://www.disgenet.org/, version: 7.0), by searching clinical symptoms of DKD and extracting the corresponding gene sets. Finally, all the genes obtained from the above three databases were merged and the redundancy was removed.

\section{Functional enrichment analyses}

Functional enrichment analyses of CRT putative targets were performed using the DAVID (Database for Annotation, Visualization and Integrated Discovery, https:// david.ncifcrf.gov/tools.jsp, version: 6.7) based on the HPO data and the KEGG pathway data (Kyoto Encyclopedia of Genes and Genomes, http://www.genome.jp/ $\mathrm{kegg} /$, last updated on October 16, 2012). Only clinical symptoms and KEGG pathways with $\mathrm{p}$ values $<0.05$ (corrected using the Bonferroni method) were selected.

\section{"Disease Gene-Drug Target" interaction network construction and analysis}

"Disease Gene-Drug Target" interaction network was constructed using links between DKD-related genes and CRT putative targets according to the public database STRING (Search Tool for Known and Predicted ProteinProtein Interactions, http://string-db.org/, version: 10.0). The gene-gene interactions with combined scores higher than 0.7 were selected in this study. Then, the three topological features, including the node degree, betweenness and closeness were calculated to screen the network target with the topological importance in the networks. The nodes with high interconnections within the network were divided into different functional modules by using the Markov clustering algorithm. The networks were visualized by Cytoscape software (version: 3.8.0).

\section{In vivo experimental validation \\ Ethics statement}

The in vivo experimental validation with animals were performed in the Institute of Chinese Materia Medica, China Academy of Chinese Medical Sciences, Beijing, China. All experimental protocols were approved by the 
Research Ethics Committee of the Institute of Chinese Materia Medica (No. IACUC-G16045). Animal experiments were carried out in accordance with the guidelines and regulations for the care and use of laboratory animals of the Center for Laboratory Animal Care, China Academy of Chinese Medical Sciences, Beijing, China.

\section{Animals and treatment}

Male Sprague-Dawley (SD) rats $(\mathrm{n}=48,190-210 \mathrm{~g}$ in weight) were purchased from Guangdong Medical Laboratory Animal Center (production license no. SCXK 2013-0002, Guangzhou, China). All rats were kept under specific pathogen-free conditions, with a constant temperature of $24 \pm 1{ }^{\circ} \mathrm{C}$ in a 12 -h light/12-h dark cycle room and adlibitum water and food access. Prior to the experiments, the rats were allowed a 1-week acclimatization period. A total of $48 \mathrm{SD}$ rats were randomly divided into normal control group $(n=8)$ and model group $(n=40)$ and provided adlibitum access to standard rodent chow and HFD, respectively. The model was established with HFD (60\% energy from fat, 20\% carbohydrate and 20\% protein) (\# D12492, Research Diets, Inc., NJ, USA). After 4 weeks of dietary manipulation, HFD-fed rats were injected intraperitoneally with $35 \mathrm{mg} / \mathrm{kg}$ Streptozotocin (STZ, freshly dissolved in precooling citrate buffer solution, pH 4.5, \#V900890, Sigma-Aldrich, St. Louis, MO, USA) twice a week. A week after STZ injection, rats with blood glucose levels over $11.1 \mathrm{mmol} / \mathrm{L}$ were divided into the DKD group $(\mathrm{n}=8)$, Metformin (\#PHR1084, Sigma-Aldrich, St. Louis, MO, USA) group (135 mg/ $\mathrm{kg}, \mathrm{n}=8$ ), CRT (Pharmaceutical Factory of the Chongqing Academy of Chinese Materia Medica, Chongqing, China) low-dosage group (CRT-L, $145 \mathrm{mg} / \mathrm{kg}, \mathrm{n}=8$ ), CRT medium-dosage group (CRT-M, $290 \mathrm{mg} / \mathrm{kg}, \mathrm{n}=8$ ), CRT high-dosage group (CRT-H, $580 \mathrm{mg} / \mathrm{kg}, \mathrm{n}=8$ ). The normal control group rats $(\mathrm{CON})$ were injected with the solvent of STZ (precooling citrate buffer solution, $\mathrm{pH}$ 4.5). In the course of administration, the rats in the model group and the treatment group were fed with HFD until the end of the study, which was 9 weeks. The DKD rat model had a mortality rate of about $20 \%$. At the end of the experiments, the rats in different groups were all anesthetized with pentobarbital sodium, the blood was taken from the abdominal aorta, and the rats were killed by cervical dislocation. Finally, all the rats were dissected and samples were taken.

\section{Detection of biochemical indicators}

Body weight, blood glucose and urine volume of the rats were measured once a week. Blood samples were taken once a week to detect the levels of insulin, C-peptide and glycosylated hemoglobin. The experiment was terminated at the end of 63 days and the rats were fasted for
$12 \mathrm{~h}$. Then, the urine was collected by metabolic cage, and the microalbuminuria, creatinine, and $24 \mathrm{~h}$ urine protein were detected by ELISA. The levels of IL-1 $\beta$, TNF- $\alpha$, total cholesterol (TC), triglyceride (TG), low density lipoprotein (LDL-C), and high density lipoprotein (HDL-C) in serum, and the levels of IL- $1 \beta$, TNF- $\alpha$, Collagen $\mathrm{l}$ and Fibronectin in cell culture medium were all measured by ELISA kits. Detailed information of all ELISA kits is provided in Additional file 1: Table S1.

\section{Histopathological evaluation}

Renal tissues obtained from different groups were stained with $\mathrm{H} \& \mathrm{E}$, periodic acid- Schiff (PAS), MASSON, and periodic acid silver methenamine (PASM) staining to observe the histopathological changes. The methods were the same as our previous studies or manufacturer's instructions [19].

\section{Immunohistochemical staining}

Immunohistochemical staining was conducted using an UltraSensitive SP (rabbit/mouse) immunohistochemistry (IHC) kit (\# KIT-9706, KIT-9701, KIT-9709; MX Biotechnologies, Fuzhou, China), which contained endogenous peroxidase blocking solution, serum, secondary antibody, streptavidin-peroxidase, and diaminobenzidine (DAB) substrate-chromogen (DAB-0031, MX Biotechnologies, Fuzhou, China). The Collagen 1 (dilution 1:800, \#PAB46098, Bio-Swamp, Wuhan, China) and Fibronectin (dilution 1:800, \# PAB46097, Bio-Swamp, Wuhan, China) antibodies were used. The rest of the experimental methods, the intensity of dyeing score and quantitative methods were the same as our previous studies [19].

\section{Immunofluorescence assay}

Immunofluorescence analyses were also carried out according to our previous description [19]. The antibodies were NF-KB (\#14,220-1-AP, Proteintech, IL, USA) and p-NF-кB (\#YP0191, Immuno Way, TX, USA).

\section{In vitro experimental validation}

The Human proximal tubular epithelial cell line (HK-2) (\# GDC0152, China Center for Type Culture Collection, Wuhan, China) was used for in vitro experimental validation. HK-2 cells were maintained in Dulbecco's modified Eagle medium media (DMEM, \#SH30022-01B, Thermo Scientific/HyClone, USA) supplemented with low glucose $(5.6 \mathrm{mmol} / \mathrm{L})$ and $10 \%$ fetal bovine serum (FBS) in a humidified $5 \% \mathrm{CO}_{2}$ incubator at $37{ }^{\circ} \mathrm{C}$. Trypsin was used to digest the HK-2 cells after being grown to $80-90 \%$. Moreover, the cells were added to the 6 -well plate with the density of $1.5 \times 10^{5}$ cells per well. After the cells were adhered to the wall, the medium containing $0.5 \%$ serum was replaced and cultured for $24 \mathrm{~h}$, so that the cells were 
in a resting phase. Then, HK-2 cells were divided into the following groups: (1) control group (CON, D-glucose $5.6 \mathrm{mmol} / \mathrm{L}$ ), (2) high glucose group (HG, D-glucose $30 \mathrm{mmol} / \mathrm{L}$ ), (3) CRT low-dosage group (CRT-L, $10 \mu \mathrm{g} /$ $\mathrm{mL}$ ), (4) CRT high-dosage group (CRT-H, CRT $50 \mu \mathrm{g} /$ $\mathrm{mL}$ ), (5) Dapagliflozin group (DAP, $10 \mu \mathrm{mol} / \mathrm{L}$ ). All treatment groups were cultured in high glucose medium (D-glucose $30 \mathrm{mmol} / \mathrm{L}$ ). After $48 \mathrm{~h}$ of coculture, the supernatant and cells were collected for further analysis.

\section{Western blot analysis}

Protein expression levels of PI3K, p-PI3K, AKT, p-AKT, NFkB and p-NF-кB (р65) in renal tissues and cells of different groups were detected by western blot analysis according to our previous studies [19-21]. The detailed information about the antibodies is as follows: PI3K (\#4249, CST, MA, USA), p-PI3K (\#bs-3332R, Bioss Inc, Beijing, China), AKT (\#ab89402, abcam, MA, USA), p-AKT (\#ab81283, abcam, MA, USA), NF-KB (p65) (\#YM3111, Immuno Way, TX, USA), р-NF-кB (р65) (\#YP0191, Immuno Way, TX, USA), and GAPDH (\# 2118, CST, MA, USA).

\section{Statistical analyses}

All experiments in the current study were performed in triplicate. Statistical analyses were performed using GraphPad Prism (version 7.0). Data are shown as the mean $\pm S D$, and were analyzed by one-way ANOVA followed by a least significant difference (LSD) test. P values $<0.05$ were considered to be significant.

\section{Results}

\section{Pharmacological potentials of CRT against DKD}

A total of 672 putative target genes of CRT were predicted as summarized in Additional file 1: Table S2. Then, we collected 3472 DKD-related genes from HPO, DisGeNet and Drugbank databases (Additional file 1: Table S3), of which 390 were putative targets of CRT. According to the enrichment analysis, the putative targets of CRT were significantly associated with several clinical symptoms of DKD, such as pollakiuria, diabetic neuropathy, inflammation, diabetic retinopathy and hypertensive disease, etc. (all $\mathrm{p}<0.05$, Additional file 1: Table S4). In terms of functional modules, the putative targets of CRT were involved into immune system regulation, substance metabolism, energy synthesis and decomposition, as well as hormone synthesis and metabolism (all $\mathrm{p}<0.05$, Additional file 1: Table S5). The above data imply that CRT might have potentials to reverse the pathological changes of DKD, which promoted us to explore its pharmacological mechanisms.

\section{CRT alleviates DKD mainly by reversing the immune-inflammatory imbalance}

"Disease Gene-Drug Target" interaction network was constructed with 23,923 links among 561 CRT targets and 2851 DKD-related genes. After calculating the network topological features, we identified a total of 231 candidate targets of CRT against DKD (Additional file 1: Table S6), which were significantly enriched in five functional modules, including the regulation of immuneinflammatory response, alleviation of renal basement membrane lesions, regulation of abnormal renal hemorheology, regulation of nervous system and energy metabolism (Fig. 1 and Additional file 1: Table S7). Notably, the regulation of immune-inflammatory module was the most enriched functional module by the candidate targets of CRT. Therefore, we would like to perform in vivo and in vitro experiments to verify the immune modulatory and anti-inflammatory actions.

\section{CRT effectively reduces blood glucose, insulin and alleviates lipid metabolic disturbance in DKD rats}

Compared with the normal control rats, DKD rats induced by HFD/STZ were featured as the reduced body weights, increased urine volume and blood glucose, which were effectively reversed by the administration of CRT at 2 weeks (Fig. 2A). In addition, the significant elevation in the serum levels of insulin and $\mathrm{C}$-peptide were observed in DKD rats from 6 to 9 weeks, and fell back to normal after the administration of CRT with high dose (Fig. 2B). Glycosylated hemoglobin may accurately reflect the degree of long-term blood glucose control, and may be the gold standard for blood glucose control in patients with diabetes [22]. As shown in Fig. 2C, a significant increase in glycosylated hemoglobin level was observed in the DKD rats, which was reversed by the administration of CRT. It is considered that the accumulation of free lipids in glomeruli and tubules leads to renal lipotoxicity, which in turn leads to tubulointerstitial fibrosis [23]. We further detected the serum levels of lipid metabolism indicators in different groups, the administration of CRT with various dosages significantly reduced the serum levels of TC, TG, LDL-C and elevated that of HDL-C (Fig. 2D).

\section{CRT effectively improves renal function, extracellular matrix deposition and ameliorates inflammatory reaction in DKD rats and HK-2 cells}

As shown in Fig. 2E, DKD rats exhibited the elevated kidney index, $24 \mathrm{~h}$ urine protein, urinary microalbuminuria and creatinine levels, and the ratio of urinary albumin to creatinine (UACR), which were all significantly decreased following the administration of CRT with high dosage. 


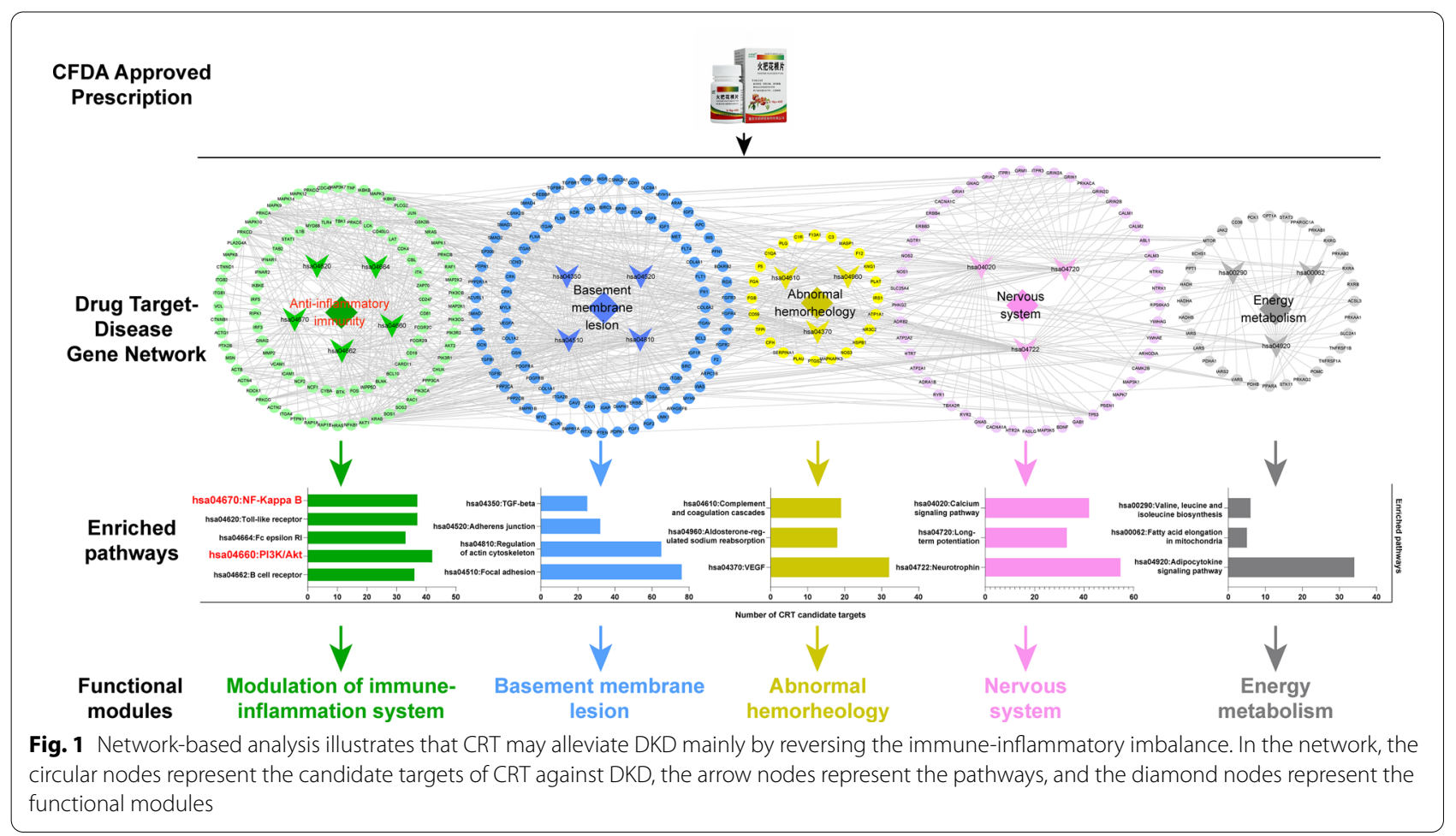

Regarding to the histopathological changes of the kidney tissues in different groups, Fig. 3A showed the lobulated glomeruli, severely atrophied glomerular vessels (blue arrow), and vacuolar degeneration cytoplasm (red arrow), inflammatory infiltration (black arrow) in DKD rats. In addition, PAS staining showed that the mesangial matrix of kidneys was significantly increased. Consistently, PASM staining of glomeruli revealed the slightly thickened basement membrane, and Masson staining revealed the slightly increased interstitial fibrosis (Fig. 3B, C). In contrast, the administration of CRT with high dose for 4 weeks effectively ameliorated the histopathological changes in diabetic kidney $[24,25]$. Similar results were found in MET treatment group. All these results indicate that CRT may improve the deterioration of renal function and structural changes in DKD rats.

Since the alterations of extracellular matrix (ECM) are one of the key pathological modifications of DKD [26], we also examined the expression patterns of profibrotic molecules fibronectin and collagen I proteins using immunohistochemistry. As shown in Fig. 3D, E, the administration of CRT significantly attenuated the enhanced immunoreactive intensity and reduced the increased number of positive immunoreactive cells of both fibronectin and collagen I in DKD rats (all $\mathrm{P}<0.05$ ). Consistently, the expression of fibronectin and collagen I in HK-2 cells treated by high glucose showed the same trend (Fig. 3F). Moreover, the serum levels of TNF- $\alpha$ and
IL-1 $\beta$ in DKD rats were significantly higher than the normal rats, but were markedly reduced in the drug treatment group. Consistently, the expression of TNF- $\alpha$ and IL- $1 \beta$ in HK- 2 cells treated by high glucose showed the same trend (Fig. 5A, B).

\section{CRT inhibits the activation of PI3K/AKT/NF-KB signaling in vivo and in vitro}

According to our network analysis, the major network targets of CRT against DKD were all significantly enriched into PI3K/AKT/NF-KB pathway (Fig. 1), which may play a pivotal role in regulating inflammatory response of various diseases. Consistently, the results of our western blot analyses showed that the expression of $\mathrm{p}-\mathrm{PI} 3 \mathrm{~K}, \mathrm{p}-\mathrm{AKT}$, and $\mathrm{p}-\mathrm{NF}-\mathrm{KB}$ proteins, as well as the ratios of $\mathrm{p}$-PI3K/total PI3K, $\mathrm{p}-\mathrm{AKT} /$ total AKT and p-NF- $\kappa \mathrm{B} /$ total NF- $\mathrm{\kappa B}$ in renal tissues of DKD rats and high glucose-induced HK-2 cells were significantly increased comparing with the normal controls. In contrast, CRT effectively reduced the expression levels of $\mathrm{p}-\mathrm{PI} \mathrm{K}, \mathrm{p}-\mathrm{AKT}$ and $\mathrm{p}-\mathrm{NF}-\mathrm{KB}$ proteins, as well as the ratio of phosphorylation to total proteins both in vivo (Fig. 4A, B) and in vitro (Figs. 5C, D and 6). Similarly, our immunofluorescence assay further showed the marked NF- $\mathrm{KB}$ (p65) translocation into the nucleus in renal tissues of DKD rats, and the situation was reversed after the administration of CRT (Fig. 4C, D). 


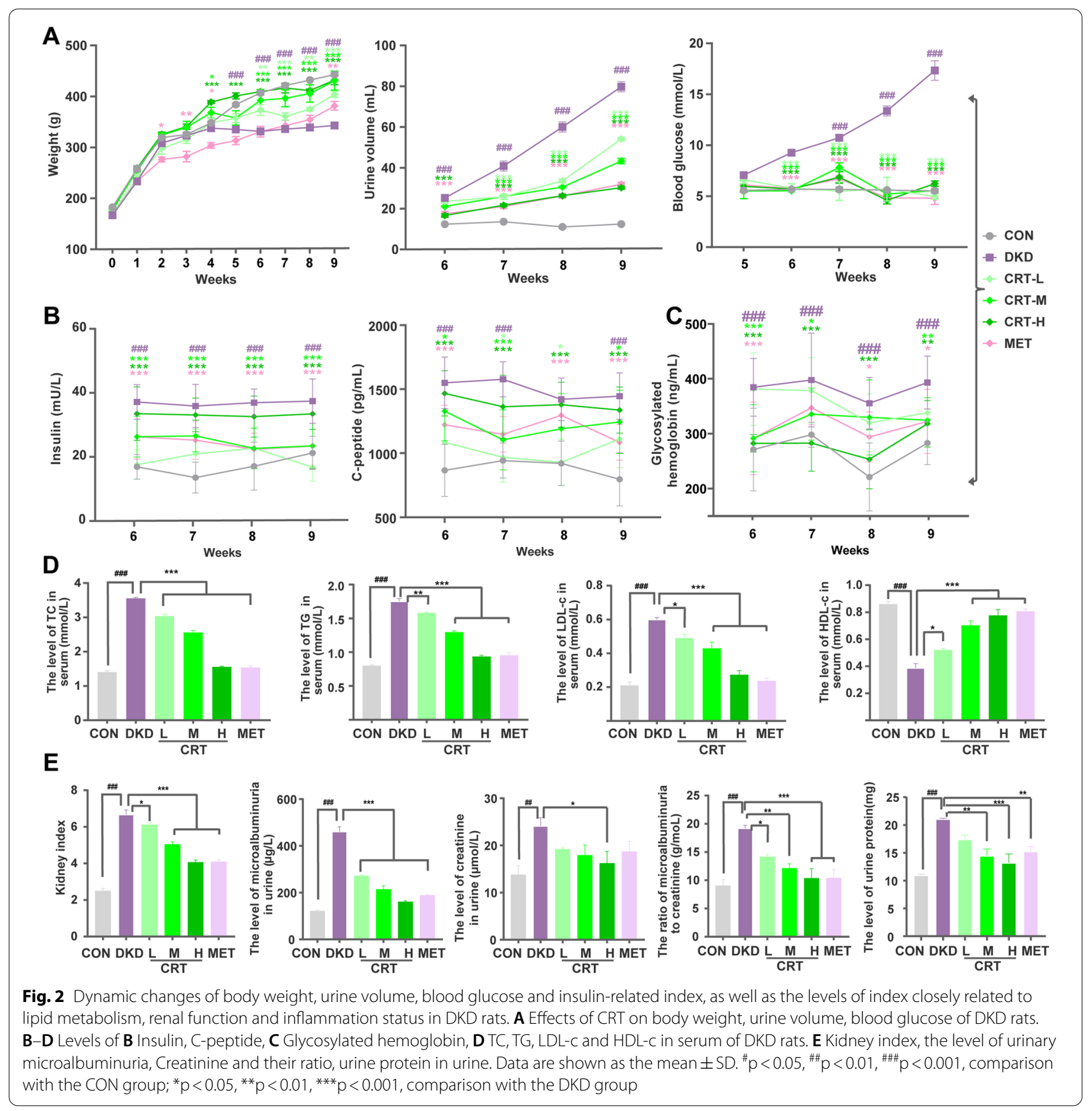

(See figure on next page.)

Fig. 3 Effects of CRT on the histopathological changes of kidney examined by H \& E, PAS, PASM, MASSON stainings, and on the expression patterns of the ECM markers in kidney tissues of DKD rats detected by immunohistochemistry. A Representative images of the kidney tissues in different groups examined by H \& E staining. Original magnification, $\times 200,400$. Scale bars represent 100, $50 \mu \mathrm{m}$. Red boxes mark the areas with typical pathological changes. B Representative images of the kidney tissues in different groups examined by PAS, PASM, MASSON stainings. Original magnification, $\times 400$. Scale bars represent $50 \mu \mathrm{m}$. C Quantitative analyses of PAS, PASM and Masson stainings. D Expression patterns of Collagen I and Fibronectin in the kidney tissues of different groups examined by immunohistochemistry. Original magnification, $\times 400$. Scale bars represent $50 \mu \mathrm{m}$. E Semiquantitative data of immunoreactivities of fibronectin and collagen I proteins in the kidney tissues of different groups, respectively. $\mathbf{F}$ Expression levels of fibronectin and collagen I proteins in HK-2 cells treated by high glucose detected by ELISA. Data are shown as the mean \pm SD. ${ }^{\#} \mathrm{p}<0.05,{ }^{\# \#} \mathrm{p}<0.01, \# \# \mathrm{n}<0.001$, comparison with the CON group; ${ }^{*} \mathrm{p}<0.05,{ }^{* *} \mathrm{p}<0.01,{ }^{* * *} \mathrm{p}<0.001$, comparison with the DKD group 


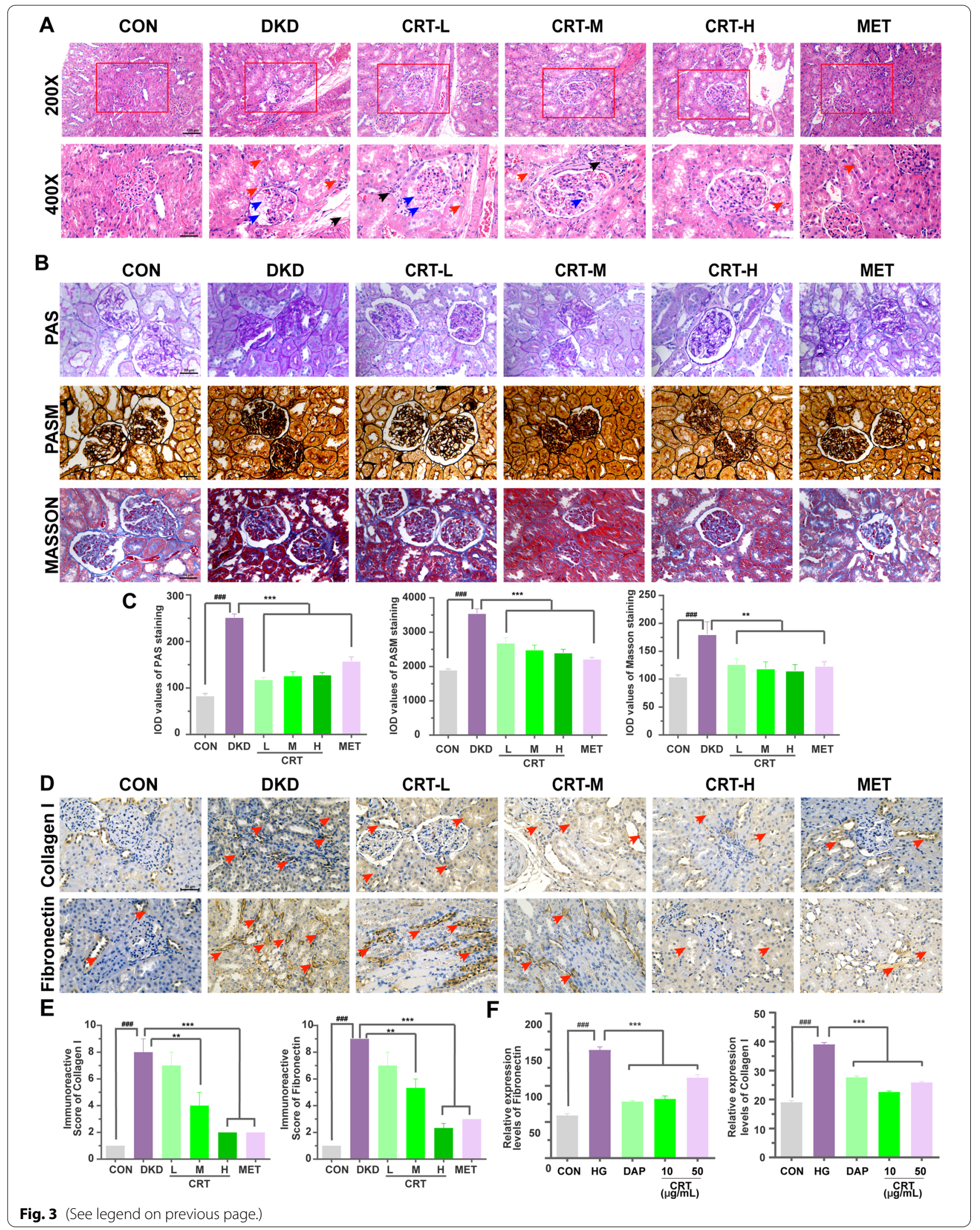




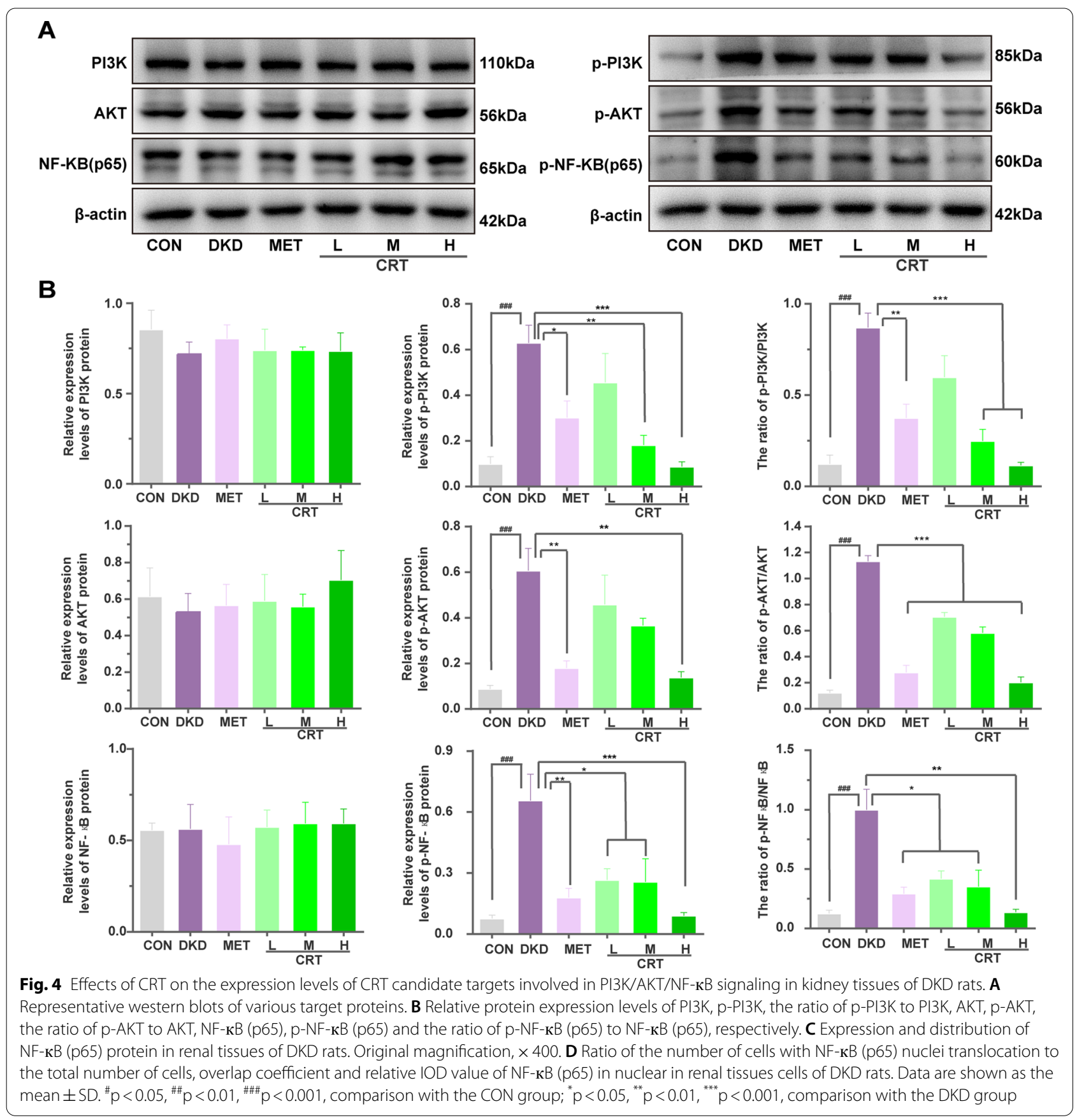

\section{Discussions}

The immune-inflammation imbalance has been indicated to promote the occurrence and the progression of DKD, implying that drugs with immune-modulatory and/or anti-inflammatory actions may be promising candidates for the treatment of this disease [27]. However, clinical trials of anti-inflammatory agents with favorable short-term outcomes are only just beginning to be published, and it will take a long time for the large-scale use of the corresponding drugs [9]. TwHF is used in the treatment of a wide spectrum of inflammatory and autoimmune diseases due to its conspicuous anti-inflammatory and immunosuppressive effects [13, 20, 21]. In the current study, we focused on the TwHFbased Chinese patent prescription CRT, which have been indicated to exert good immunomodulatory and anti-inflammatory effects in previous studies [28]. Following a series of computational analyses on "Disease 
C
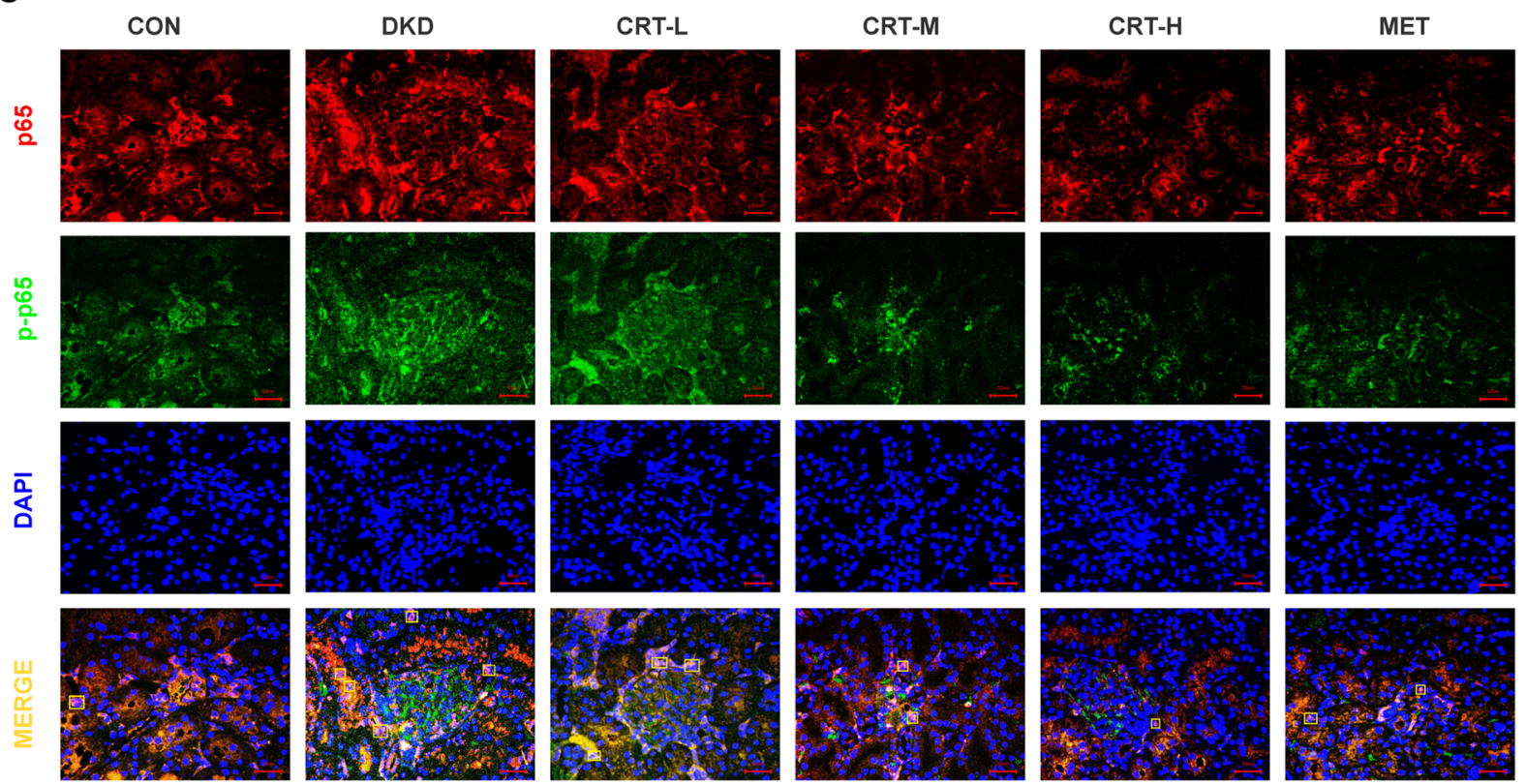

D
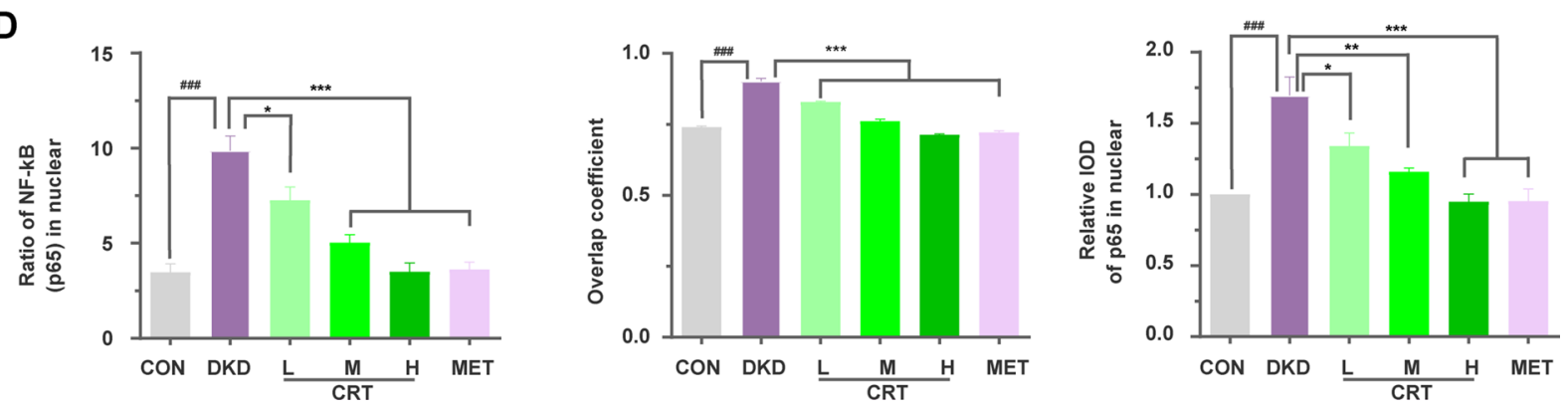

Fig. 4 continued

Gene-Drug Target" interaction network, our data firstly showed that the candidate network targets of CRT against DKD might be significantly associated with pathways related to immune-inflammation and remission of renal basement membrane lesion. Both the key network target identification and functional module analysis revealed that the inflammation-immune mediated by PI3K/AKT/NF-kB signal may be associated with the pharmacological properties of CRT to ameliorate DKD. Experimentally, CRT effectively reduced the elevated level of blood glucose, ameliorated kidney function and the renal histopathology abnormality, including inflammation, abnormal lipid metabolism, and glomerular structural damages in HFD/STZinduced DKD rats. Furthermore, the above efficacy of CRT is closely related to the activation of PI3K, AKT and NF-kB proteins, the reduction of nuclear accumulation of NF-kB protein, and the serum levels of downstream cytokines, such as IL- $1 \beta$ and TNF- $\alpha$, which were in line with the in vitro findings based on HK-2 cells induced by high glucose. These findings highlight the main pharmacological properties of CRT against

(See figure on next page.)

Fig. 5 Effects of CRT on the expression levels of CRT candidate targets involved in PI3K/AKT/NF-KB signaling, and the distribution of NF- $\mathrm{B}$ ( $\mathrm{p65}$ ) protein in HK-2 cells treated with High Glucose. A, B Expression levels of IL-1 $\beta$ and TNF-a in serum and HK-2 cells treated with high glucose detected by ELISA. C Representative western blots. D Relative protein expression levels of PI3K, p-PI3K, the ratio of p-PI3K to PI3K, AKT, p-AKT, the ratio of р-AKT to AKT, NF-кB (p65), p-NF-кB (p65), the ratio of p-NF-кB (p65) to NF-кB (p65) in HK-2 cells treated with High Glucose. Data are shown as the mean \pm SD. $\# p<0.05,{ }^{\#} p<0.01$, \#\#\# $p<0.001$, comparison with the CON group; ${ }^{*} p<0.05$, ${ }^{* *} p<0.01,{ }^{* * *} p<0.001$, comparison with the HG group 
A

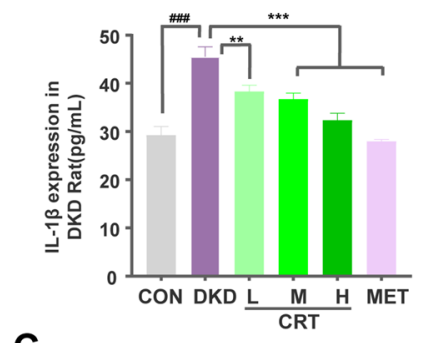

C
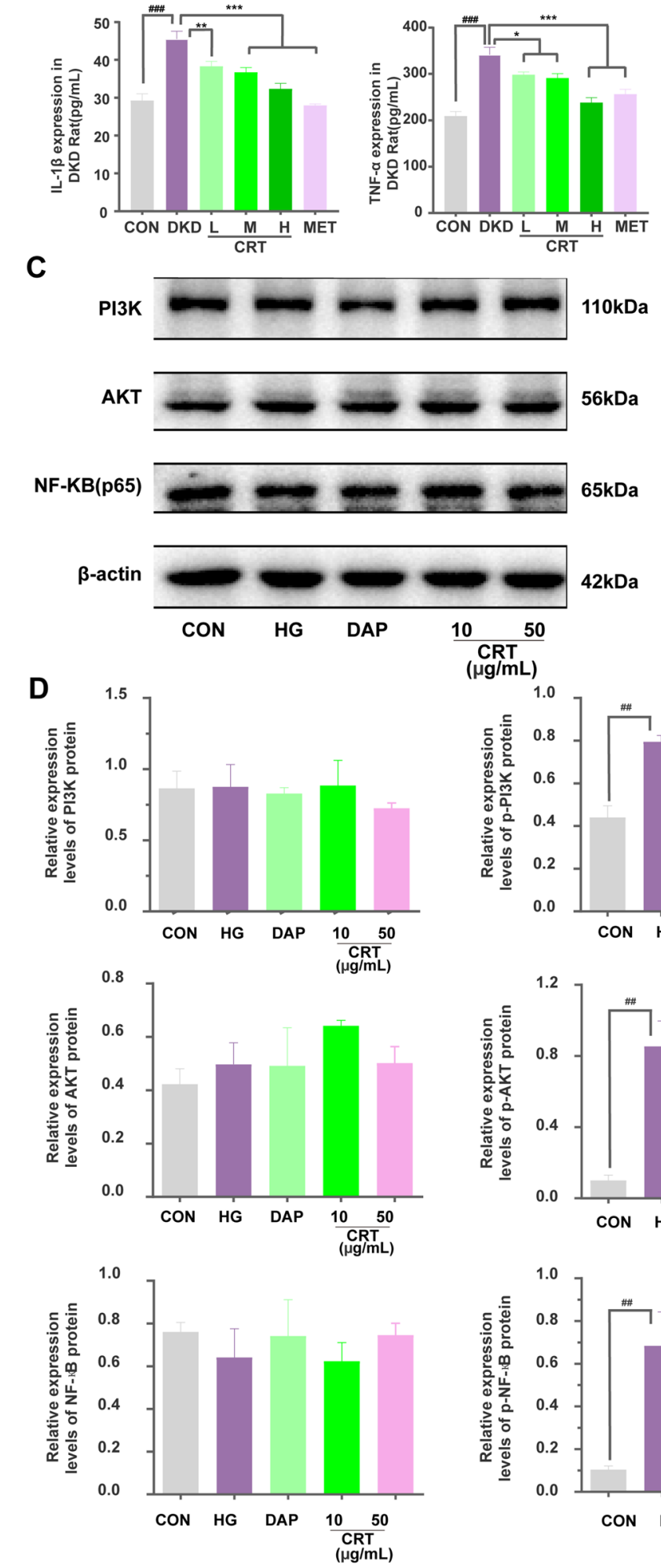

B
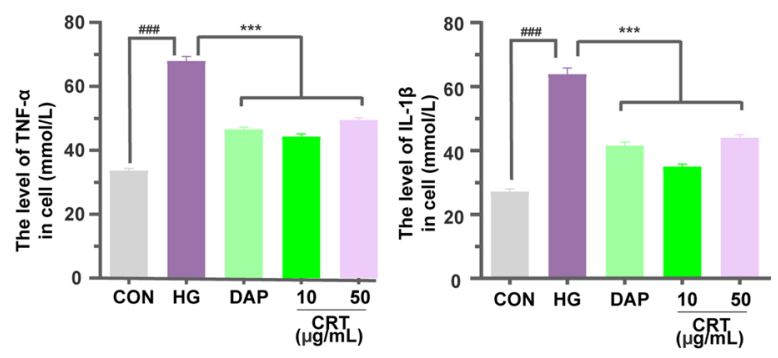

$\left(\mathrm{mg}^{\mathrm{CRT}} \mathrm{mL}\right)$
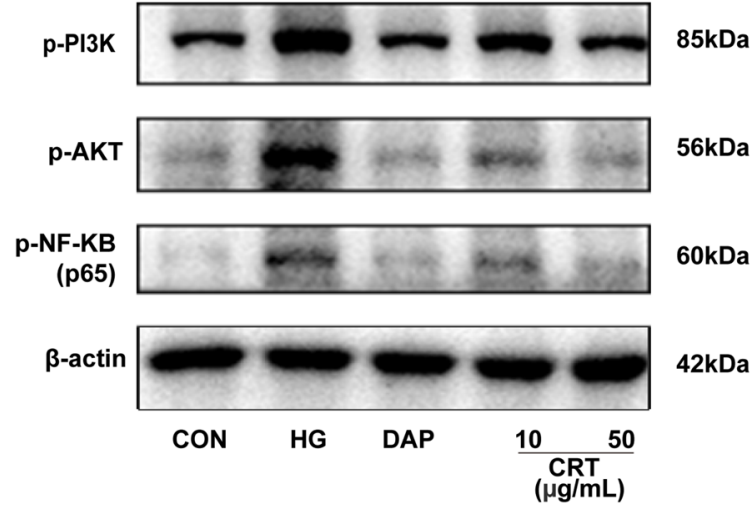

42kDa

Fig. 5 (See legend on previous page.)
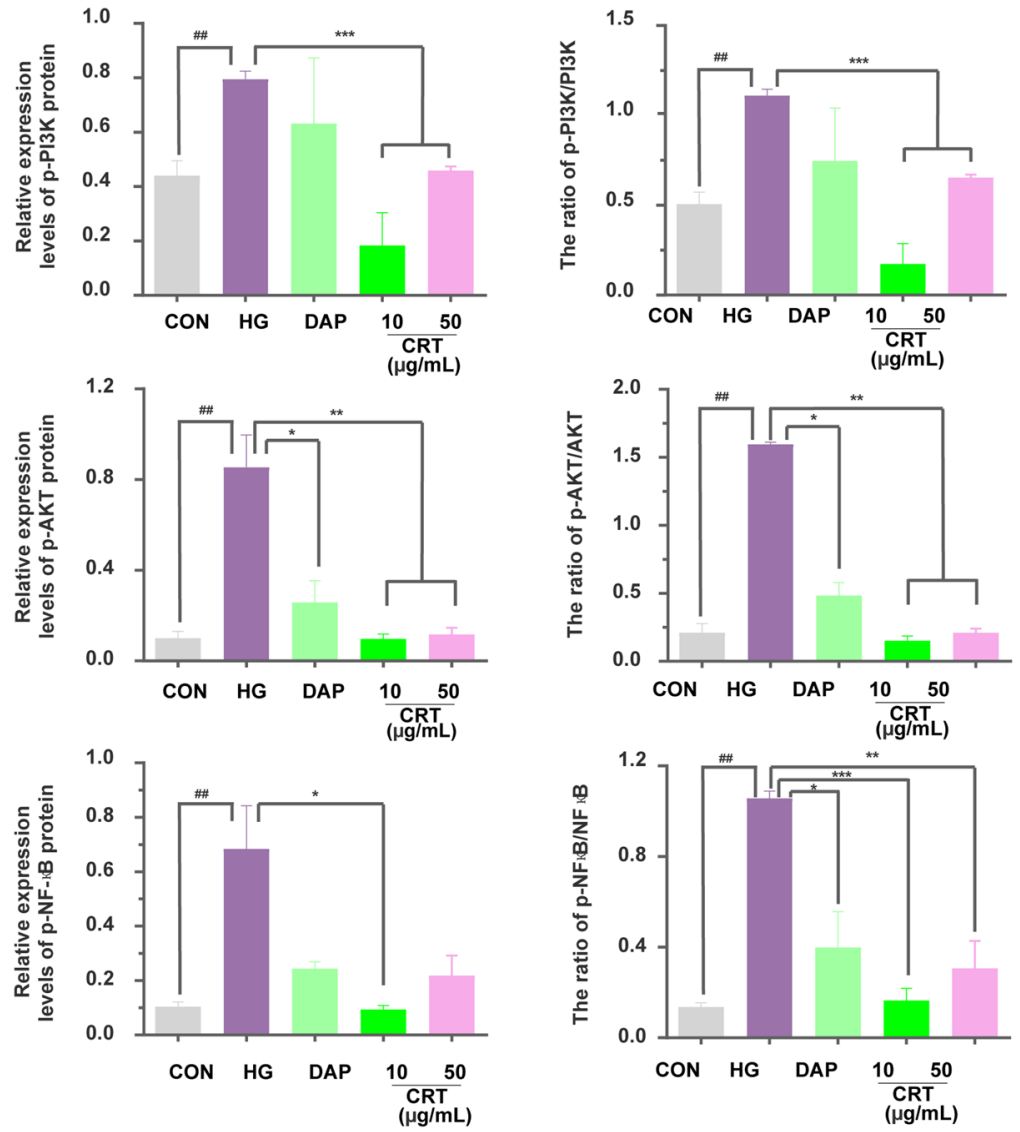

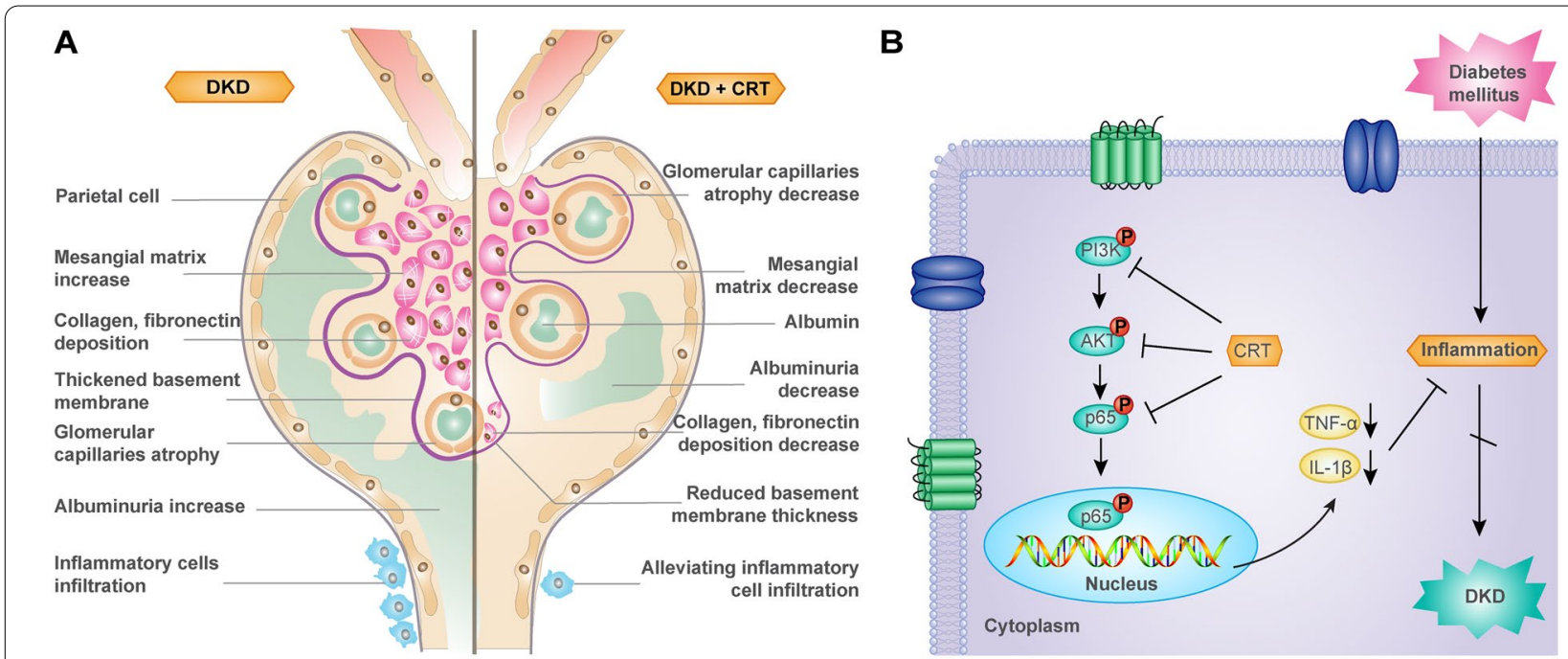

Fig. 6 Illustration of the pharmacological mechanisms underlying the therapeutic effects of CRT against DKD. A Histopathological changes of DKD kidney before and after the administration of CRT. B The mechanism of CRT-induced inhibition of inflammatory cytokine production

DKD by reversing the imbalance of immune-inflammation system during the occurrence and development of this disease.

The pathogenesis of DKD involves inflammation, fibrosis and abnormal lipid metabolism [9]. Persistent hyperglycemia destroys the glomerular filtration barrier, leading to glomerular structural damage, resulting in urinary protein or albumin leakage, and which, in turn further aggravates the progression of DKD [29]. Mesangial matrix expansion and thickening of the glomerular basement membrane are the main hallmarks of DKD [30]. Both inflammatory cytokines and high glucose can stimulate tubular epithelial cells to produce collagen I and fibronectin, which are the markers of ECM. Excessive accumulation of ECM in mesangium and glomerular basement membrane may accelerate tubulointerstitial fibrosis [31, 32]. Consistently, our in vivo experiments observed the enhanced albuminuria, urinary protein excretion (UACR), aggravated mesangial matrix expansion, basement membrane thickening and extracellular matrix deposition in glomerular, and worsening renal tissue inflammation and interstitial fibrosis, as well as elevated lipid metabolism in DKD rats. In contrast, the administration of CRT effectively reversed the above pathological changes and attenuated the development of DKD. Chronic inflammation and fibrosis play important roles in the occurrence and development of complications of DKD [25]. Like renal interstitial fibrosis, DKD may be stimulated by persistent hyperglycemia and inflammatory factor [33]. It has been reported that TNF- $\alpha$ and IL- $1 \beta$ may induce proximal tubular epithelial cells to secrete ECM markers (type I collagen and fibronectin), which accelerates renal tubular interstitial fibrosis [34].

NF-kB signaling is the key pathway to tissue inflammation, because NF-kB may control the expression of inflammatory cytokines, chemokines and adhesion molecules, all of which play a key role in controlling inflammation [20, 35-38]. In addition, the inhibition of NF-kB may effectively alleviate renal inflammation and fibrosis caused by persistent hyperglycemia in DKD [25]. In the current study, the activation of NF-kB protein and the levels of TNF- $\alpha$ and IL- $1 \beta$ were positively associated with the renal inflammatory damage and extracellular matrix deposition in both DKD rats and HK-2 cells treated by high glucose, which were also consistent with previous reports [25]. Moreover, network analysis results showed that the upstream signaling of inflammation such as PI3K/Akt, Toll-like receptor 4/2, Tim 3, HMBG1, and so on, may be the important mechanism by which CRT alleviates inflammatory damage in DKD. Recent evidence show that PI3K/Akt signaling pathways may be critical in the modulation of ECM expression and tubule-interstitial fibrosis in chronic renal diseases. Even though, whether PI3K/Akt signaling may be associated with the anti-inflammatory effects of CRT is still unclear. Herein, we focused on the upstream signaling of NF-kB signaling-mediated inflammatory pathway, PI3K/Akt signaling, and found that the persistent hyperglycemia activated PI3K/Akt pathway, which promoted the activation of NF- $\mathrm{KB}$ signaling and the release of inflammatory cytokines, subsequently leading to massive proteinuria, tubulointerstitial inflammatory infiltration, mesangial matrix expansion and basement membrane thickening in 
DKD rats. On the contrary, CRT efficiently reversed the above pathological changes by suppressing the activation of PI3K/AKT/NF-kB pathway and inhibiting the translocation of p65 into the nucleus.

\section{Conclusion}

Our findings provide the evidences that the TwHF-based prescription, CRT may be the promising candidate drugs for the treatment of DKD via reversing the imbalance of Immune-Inflammation system which is mediated by PI3K/AKT/NF-KB/IL-1 $\beta /$ TNF- $\alpha$ signaling during the disease progression.

\begin{abstract}
Abbreviations
ADMET: Absorption distribution metabolism excretion toxicity; ANOVA: Analysis of variance; AKT: Protein kinase B; CFDA: National medical products administration; CON: Control; CRT: Colquhounia root tablets; CRT-L: Colquhounia root tablets-low-dosage group; CRT-M: Colquhounia root tablets-mediumdosage group; CRT-H: Colquhounia root tablets—high-dosage group; DAB: Diaminobenzidine; DAP: Dapagliflozin; DAVID: Database for annotation, visualization andintegrated discovery; DKD: Diabetic kidney disease; ESRD: End stage renal disease; ELISA: Enzyme linked immunosorbent assay; ETCM: Encyclopedia of traditional chinese medicine; FDA: Food and drug administration; GAPDH: Glyceraldehyde-3-phosphate dehydrogenase; HDL-C: High density lipoprotein — cholesterol; H \& E: Hematoxylin and eosin; HFD: High fat diet; HK-2: Human kidney-2; HPO: Human phenotype ontology; IHC: Immunohistochemistry; IL-1 $\beta$ : Interleukin-1 $\beta$; KEGG: Kyoto encyclopedia of genes and genomes; LDL-C: Low density lipoprotein—cholesterol; LSD: Least significant difference; NF-KB: Nuclear factor—kappa B; PAS: Periodic acid- schiff; PASM: Periodic acid-silver metheramine; PI3K: Phosphoinositide 3-kinase; QED: Quantitative estimate of drug-likeness; SD: Sprague dawley; STZ: Streptozotocin; TC: Total cholesterol; TCM: Traditional Chinese medicine; TG: Triglyceride; TNF-a: Tumour necrosis factor-a; TWHF: tripterygium wilfordii Hookf.
\end{abstract}

\section{Supplementary Information}

The online version contains supplementary material available at https://doi. org/10.1186/s13020-021-00563-7.

Additional file 1: Table S1. Includes detailed information of all enzymelinked immunosorbent assay kits. Table S2. Includes 672 putative target genes of CRT predicted using ETCM database and TCMIP v2.0 platform. Table S3. Includes 3472 DKD-related genes from Human Phenotype Ontology (HPO), DisGeNet and Drugbank databases. Table S4. Includes enrichment results of clinical symptoms of 390 CRT putative targets. Table S5. Includes enrichment results of functional modules of 390 CRT putative targets. Table S6. Includes 231 candidate targets of CRT against DKD in "Disease Gene-Drug Target" interaction networks based on calculated network topological features. Table S7. Includes enrichment results of functional modules of 231 CRT candidate targets.

\section{Acknowledgements}

Not applicable.

\section{Authors' contributions}

$\mathrm{YZ}$ and NL engaged in study design and coordination, material support for obtained funding, and supervised the study. YZ performed network analysis, designed the experimental validation. ZM performed the experiments, wrote and revised the manuscript. $Y L$ and $C L$ performed most of the experiments and statistical analyses. All authors read and approved the final manuscript.

\section{Funding}

This study was supported by grants from Scientific and technological innovation project of China Academy of Chinese Medical Sciences (No.
CI2021A03807), National Natural Science Foundation of China (No. 81974526), Beijing Municipal Natural Science Foundation (No. 7212186) and the Technology R\&D Project of Institute of Chinese Materia Medica, China Academy of Chinese Medical Sciences.

\section{Availability of data and materials}

All supporting data are included within the main article and its Additional files.

\section{Declarations}

\section{Ethics approval and consent to participate}

The in vivo experimental validation with animals were performed in the Institute of Chinese Materia Medica, China Academy of Chinese Medical Sciences, Beijing, China. All experimental protocols were approved by the Research Ethics Committee of the Institute of Chinese Materia Medica (No. IACUC-G16045). Animal experiments were carried out in accordance with the guidelines and regulations for the care and use of laboratory animals of the Center for Laboratory Animal Care, China Academy of Chinese Medical Sciences, Beijing, China.

\section{Consent for publication}

All authors have approved the publication of this submission.

\section{Competing interests}

The authors declare that there is no conflicts of interests regarding the publication of this paper.

Received: 6 October 2021 Accepted: 25 December 2021

Published online: 04 January 2022

\section{References}

1. Gallagher H, Suckling RJ. Diabetic nephropathy: Where are we on the journey from pathophysiology to treatment. Diabetes Obes Metab. 2016;18(7):641-7.

2. Shen Z, Fang Y, Xing T, et al. Diabetic nephropathy: from pathophysiology to treatment. J Diabetes Res. 2017;2017:2379432.

3. Liu WT, Peng FF, Li HY, et al. Metadherin facilitates podocyte apoptosis in diabetic nephropathy. Cell Death Dis. 2016;7(11):e2477.

4. Gheith O, Farouk N, Nampoory N, et al. Diabetic kidney disease: world wide difference of prevalence and risk factors. J Nephropharmacol. 2016:5(1):49-56.

5. Anders HJ, Huber TB, Isermann B, et al. CKD in diabetes: diabetic kidney disease versus nondiabetic kidney disease. Nat Rev Nephrol. 2018;14(6):361-77.

6. Yaribeygi $\mathrm{H}$, Atkin SL, Sahebkar A. Interleukin-18 and diabetic nephropathy: a review. J Cell Physiol. 2019;234(5):5674-82.

7. Dai H, Liu Q, Liu B. Research progress on mechanism of podocyte depletion in diabetic nephropathy. J Diabetes Res. 2017;2017:2615286.

8. Zhang L, Yang Z, Zhao Y, et al. Renoprotective effects of Gushen Jiedu capsule on diabetic nephropathy in rats. Sci Rep. 2020;10(1):2040.

9. Tang SCW, Yiu WH. Innate immunity in diabetic kidney disease. Nat Rev Nephrol. 2020;16(4):206-22.

10. Tuttle KR, Brosius FC, Adler SG, et al. JAK1/JAK2 inhibition by baricitinib in diabetic kidney disease: results from a Phase 2 randomized controlled clinical trial. Nephrol Dial Transplant. 2018;33(11):1950-9.

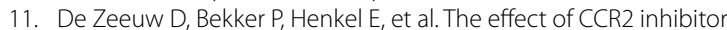
CCX140-B on residual albuminuria in patients with type 2 diabetes and nephropathy: a randomised trial. Lancet Diabetes Endocrinol. 2015;3(9):687-96

12. Heerspink HJL, Parving H-H, Andress DL, et al. Atrasentan and renal events in patients with type 2 diabetes and chronic kidney disease (SONAR): a double-blind, randomised, placebo-controlled trial. The Lancet. 2019;393(10184):1937-47.

13. Zhang Y, Mao X, Li W, et al. Tripterygium wilfordii: An inspiring resource for rheumatoid arthritis treatment. Med Res Rev. 2021;41(3):1337-74.

14. Xu HY, Zhang YQ, Liu ZM, et al. ETCM: an encyclopaedia of traditional Chinese medicine. Nucleic Acids Res. 2019;47(D1):D976-82. 
15. Wang $L$, Fan $L$, Yin M. Effect of colquhounia root tablet on type 2 diabetic nephropathy and the levels of serum HGF and urine TGF- $\beta 1$. Chin J Integr Tradit West Med. 2015; 24(19): 2057-9+82.

16. Wang X, Pan Z, Gao R. Influence of Huoba Huagen tablets combined with valsartan on renal function and lipid metabolism in patients with diabetic nephropathy. Chin J Tradit Chin Med Pharm. 2016;14(11):1860-2.

17. Wang $L, Y u J$, Luo $M$, et al. The effect of Huobahuagen tablets in intervening diabetic nephropathy in clinical stage and the influence on the level of serum HGF. J Nangjing Univ Tradit Chin Med. 2015;31(5):424-7.

18. Li M. Analysis of 70 clinical cases of massive proteinuria of diabetic nephropathy under the treatment of Huoba Huagen tablets. Chin Hosp Pharm J. 2014;34(20):1762-4.

19. Zhang $Y$, Mao $X$, Chen $W$, et al. A discovery of clinically approved formula FBRP for repositioning to treat HCC by inhibiting PI3K/AKT/NF-kappaB activation. Mol Ther Nucleic Acids. 2020;19:890-904.

20. Zhang Y, Wang $X, L i W$, et al. Inferences of individual differences in response to tripterysium glycosides across patients with Rheumatoid arthritis using a novel ceRNA regulatory axis. Clin Transl Med. 2020;10(6):e185.

21. Mao X, Li W, Chen W, et al. Exploring and characterizing a novel combination of paeoniflorin and talatizidine for the treatment of rheumatoid arthritis. Pharmacol Res. 2020;153:104658.

22. Ruospo M, Saglimbene VM, Palmer SC, et al. Glucose targets for preventing diabetic kidney disease and its progression. Cochrane Database Sys Rev. 2017. https://doi.org/10.1002/14651858.CD010137.pub2.

23. Alicic RZ, Rooney MT, Tuttle KR. Diabetic kidney disease: challenges, progress, and possibilities. Clin J Am Soc Nephrol. 2017;12(12):2032-45.

24. Weil EJ, Lemley KV, Fau-Yee B, Lovato T, et al. Podocyte detachment in type 2 diabetic nephropathy. Am J Nephrol. 2012. https://doi.org/10. $1159 / 000327047$

25. Yang $H$, Xie T, Li D, et al. Tim-3 aggravates podocyte injury in diabetic nephropathy by promoting macrophage activation via the NF-kappaB/ TNF-alpha pathway. Mol Metab. 2019:23:24-36.

26. Kolset SO, Reinholt FP, Jenssen T. Diabetic nephropathy and extracellular matrix. J Histochem Cytochem. 2012;60(12):976-86.

27. Rayego-Mateos S, Morgado-Pascual JL, Opazo-Rios L, et al. Pathogenic pathways and therapeutic approaches targeting inflammation in diabetic nephropathy. Int J Mol Sci. 2020. https://doi.org/10.3390/ijms21113798.

28. Hu J, Yang W, Mi B. Therapeutic effect and its mechanism of Huobahua Root Tablets on adjuvant arthritis in lewis rats. Chin J Clin Pharmacol. 2017;33(05):82-7.

29. Ma ST, Liu DL, Deng JJ, et al. Effect of arctiin on glomerular filtration barrier damage in STZ-induced diabetic nephropathy rats. Phytother Res. 2013;27(10):1474-80.

30. Ibrahim HN, Hostetter TH. Diabetic nephropathy. J Am Soc Nephrol. 1997:8(3):487-93.

31. Hu C, Sun L, Xiao L, et al. Insights into the mechanisms involved in the expression and regulation of extracellular matrix proteins in diabetic nephropathy. Curr Med Chem. 2015;22(24):2858-70.

32. Ranganathan $P$, Jayakumar C, Ramesh G. Proximal tubule-specific overexpression of netrin-1 suppresses acute kidney injury-induced interstitial fibrosis and glomerulosclerosis through suppression of IL-6/STAT3 signaling. Am J Physiol Renal Physiol. 2013;304(8):F1054-65.

33. Yoon JJ, Park JH, Kim HJ, et al. Dianthus superbus improves glomerular fibrosis and renal dysfunction in diabetic nephropathy model. Nutrients. 2019;11(3):247-58.

34. Reidy K, Kang HM, Hostetter T, et al. Molecular mechanisms of diabetic kidney disease. J Clin Invest. 2014;124(6):2333-40.

35. Yang GJ, Song YQ, Wang W, et al. An optimized BRD4 inhibitor effectively eliminates NF-kappaB-driven triple-negative breast cancer cells. Bioorg Chem. 2021;114:105158

36. Yang GJ, Wang W, Lei PM, et al. A 7-methoxybicoumarin derivative selectively inhibits BRD4 BD2 for anti-melanoma therapy. Int J Biol Macromol. 2020:164:3204-20.

37. Xia S, Zhong Z, Gao B, et al. The important herbal pair for the treatment of COVID-19 and its possible mechanisms. Chin Med. 2021;16:25.

38. García-García PM, Getino-Melián MA, Domínguez-Pimentel V, et al. Inflammation in diabetic kidney disease. World J Diabetes. 2014:5:431-43.

\section{Publisher's Note}

Springer Nature remains neutral with regard to jurisdictional claims in published maps and institutional affiliations.
Ready to submit your research? Choose BMC and benefit from:

- fast, convenient online submission

- thorough peer review by experienced researchers in your field

- rapid publication on acceptance

- support for research data, including large and complex data types

- gold Open Access which fosters wider collaboration and increased citations

- maximum visibility for your research: over $100 \mathrm{M}$ website views per year

At BMC, research is always in progress.

Learn more biomedcentral.com/submissions 